\title{
Investigations of Microtubule-associated Protein 2 Gene Expression in Spinal Muscular Atrophy
}

\author{
(1) Gamze Bora1', (1) Ceren Sucularlı2, (1) Niko Hensel 3, (1) Peter Claus3,4, (1) Hayat Erdem Yurter ${ }^{1}$ \\ ${ }^{1}$ Hacettepe University Faculty of Medicine, Department of Medical Biology, Ankara, Turkey \\ ${ }^{2}$ Hacettepe University Institute of Health Sciences, Department of Bioinformatics, Ankara, Turkey \\ ${ }^{3}$ Hannover Medical School, Institute of Neuroanatomy and Cell Biology, OE 4140, Carl-Neuberg-Str. 1, 30625, Hannover, Germany \\ ${ }^{4}$ Center for Systems Neuroscience (ZSN) Hannover, Germany
}

\begin{abstract}
Aim: Spinal muscular atrophy (SMA) is a devastating genetic disease in childhood andff is caused by the absence of functional survival motor neuron (SMN) protein, which leads to impairments of the cytoskeleton, especially in neurons. Dysregulation of actin dynamics have been linked to SMA patho mechanisms, however involvement of altered microtubule dynamics is largely unknown. In this study, we investigated differentially expressed microtubule-related genes using in vitro and in vivo SMA model systems.

Materials and Methods: By focusing on microtubule-related genes, we re-analyzed publically available gene expression arrays, which were previously performed with induced pluripotent stem cell-derived motor neurons of SMA patients and the spinal cords of SMA mice. We found altered expressions of microtubule-associated protein 2 (MAP2), which was validated by real time reverse-transcription polymerase chain reaction using the SMN knock-down NSC34 cell line and the severe SMA mouse model.

Results: We showed that the expression of MAP2 gene was significantly upregulated in both expression arrays. Upregulation was also detected in the brain and spinal cord tissues of severe SMA mice at different developmental stages.

Conclusion: Our findings suggest that microtubule regulatory proteins may be altered in SMN depleted cells and further research is needed to elucidate the contribution of dysregulated microtubule dynamics towards SMA.
\end{abstract}

Keywords: Spinal muscular atrophy, exon-array, microtubule-associated protein 2

\section{Introduction}

Spinal muscular atrophy (SMA) is an inherited neurodegenerative/neuromuscular disease and the leading genetic cause of infant mortality. The incidence of SMA is reported as 1 in 11.000 live births, however, due to a high rate of consanguinity, it is estimated to be higher in Turkey (1). SMA is characterized by the loss of alpha motor neurons in the spinal cord and progressive muscle atrophy. Since patients have different clinical phenotypes, SMA is grouped into $V$ Types (O-IV) according to the age of disease onset and achieved motor functions $(2,3)$. Type 0 refers to the most severe and the Type IV refers to the mildest form of SMA. Mutations or conversions of the Survival of motor neuron 1 (SMN1) gene are responsible for SMA and regardless of disease severity, homozygous deletion of exon 7 and 8 or exon 7 only is the most frequent mutation in patients (4-6). It has been shown that the absence of ubiquitously expressed, functional SMN protein leads to defects in both axon and dendrite growth, axonal transport and neuromuscular junction 
maturation in model systems and also patient samples (7-11). Dysregulation of F-actin dynamics have been linked to these defects due to alterations in either actinregulatory proteins such as profilin, plastin 3 , coronin $1 C$ or Rho-kinase (ROCK) signaling pathways in SMN depleted cells $(6,7,12,13)$. Although significant alterations in some microtubule-related proteins (stathmin and tau) have been shown, the contribution of altered microtubule dynamics to SMA patho mechanisms is largely unknown $(14,15)$.

Re-analysis of publicly available gene expression data is a powerful and cost-efficient technique to better understand disease mechanisms. This technique has been used to explore the molecular mechanisms of various diseases, such as different cancers $(16,17)$, osteoarthritis (18) and degenerative diseases (19). Furthermore, metaanalysis and the comparison of gene expression profiles of different species enables researchers to discover conserved molecular mechanisms (20).

Therefore, in this study, we re-analyzed human and mouse microarray gene expression data and specifically focused on genes regulating microtubule structure and function. We found that the expression of MAP2 was significantly altered in both induced pluripotent stem cell (iPSC) derived motor neurons of SMA patient and the spinal cords of SMA mice when compared to controls. MAP2 is primarily expressed in neurons and localizes to cell bodies and dendrites in mature neurons. The MAP2 protein binds to microtubules and regulates their stability. It also binds to F-actin and bundle filaments in vitro (21). Therefore, we focused on the MAP2 gene and analyzed its expression in both the SMN knock-down motor neuron like NSC34 cell line and the Taiwanese SMA mouse model (22).

\section{Materials and Methods}

\section{Human and Mouse Dataset Retrieval}

The Gene Expression Omnibus (http://www.ncbi.nlm. nih.gov/geo/, $(23,24)$ database was searched for Human Exon arrays of motor neuron samples obtained from an SMA patient and a control. A record GSE27205 (25), which was on the Affymetrix Human Exon 1.0 ST platform (HuEx-1_0-st), was identified and CEL files of iPSC-derived motor neurons from an SMA patient $(n=3, n$ is different clones from an SMA patient; GSM672172, GSM672173 and GSM672174) and a heterozygous father $(n=3, n$ is different clones from an SMA patient's father; CSM672178, CSM672179 and CSM672180) were selected and extracted from the GEO database. This article does not contain any studies with human participants performed by the authors. Informed consent wasn't obtained. Mouse microarray data GSE19674, which was on an Affymetrix Mouse Genome
430A 2.0 Array platform (Mouse430A_2), including CEL files of spinal cords of homozygous knock-out SMA mice $\left(\mathrm{n}=4, \mathrm{SMN2} 2^{++}\right.$; SMN $\Delta 7^{+/+} ; \mathrm{mSmn}^{-1-}$; FVB.Cg-Tg (SMN2*delta7) 4299Ahmb Tg (SMN2) 89Ahmb Smn1tm1Msd) and heterozygous SMN knock-out mice $\left(n=4, \mathrm{SMN}^{+/+}\right.$; $\mathrm{Smn} \Delta 7^{+/{ }^{+}}$; mSmn+/-; FVB.Cg-Tg (SMN2*delta7) 4299Ahmb Tg (SMN2) 89Ahmb Smn11tm1Msd) (26). CEL files for SMA mice spinal cord (GSM491297, GSM491298, GSM491299 and GSM491300) and heterozygous SMN knock-out mice spinal cord (CSM491293, GSM491294, GSM491295 and GSM491296) were obtained from the GEO database.

\section{Human and Mouse Gene Expression Analysis}

Affymetrix Human Exon 1.0 ST array CEL files of an SMA patient and heterozygous father, and Affymetrix Mouse Genome 430A 2.0 Array CEL files of an SMA and heterozygous SMN knock-out mouse were analyzed via the Transcriptome Analysis Console 4.0.1.36 (TAC, https://www. thermofisher.com). For the human exon array analysis, Gene Level-Core, robust multichip average (RMA)-sketch workflow was applied to create probe level summarization files. For Mouse430A 2 arrays, the RMA algorithm $(27,28)$ was used to normalize CEL files. The annotation files HuEx-1_0-st-v2.na36.hg19.transcript.csv for HuEx-1_0-st array and Mouse430A_2.na36.annot.csv for Mouse430A_2 array were used to annotate human and mouse arrays, respectively. Probe sets with ANOVA (eBayes) p-value $<0.05$ and fold change $<-1.5$ or fold change $>1.5$ were considered as differentially expressed for both human and mouse datasets.

\section{Venn Diagram Analysis}

The significantly altered genes, which are involved in microtubule structure and regulation of its dynamics, of human and mouse datasets were compared using Venny 2.1 (http://bioinfogp.cnb.csic.es/tools/venny/) (29).

\section{Cell Culture and siRNA Transfections}

Motor neuron-like murine NSC34 cells were grown in Dulbecco's modified Eagle medium (DMEM, 4.5 g/D-glucose), containing $5 \%$ fetal calf serum and $1 \%$ penicillin/streptomycin at $37{ }^{\circ} \mathrm{C}, 5 \% \mathrm{CO}_{2}$. The cells were transfected with siRNA against murine SMN (5'-CAGAAGUAAAGCACACAGCAA-3') or scrambled control siRNA (5'-GCGCAAAUAAACCGAAAGACA-3') by using OptiMeM (Thermo Scientific) and Lipofectamine 2000 (Invitrogen) in differentiation medium, containing 1\% FSC for 72 hours. The SMN knock down efficiency of NSC34 cells was about $80 \%$, which was routinely tested by Western blot.

\section{RNA Isolation and Real Time RT-PCR}

Total RNA was isolated from NSC34 cells by the RNeasy mini kit (Qiagen) using the manufacturer's protocol. Spinal 
cord ( $\mathrm{p} 1, \mathrm{p} 5$ and $\mathrm{p} 8$ ) and brain ( $\mathrm{p} 8)$ RNA samples of severe the SMA Taiwanese mice model (FVB.Cg-Tg (SMN2) 2Hung SMN1tm1Hung/J (22) (Jackson Laboratory) and heterozygous control littermates, which were previously isolated and stored at $-80^{\circ} \mathrm{C}$, according to German animal welfare regulations (breeding approved by the Lower Saxony State Office for Consumer Protection and Food Safety (LAVES, reference number 15/1774 LAVES). The numbers of mice used from different developmental stages are provided in the figure legends. cDNA synthesis was performed as previously reported (30). Briefly, 2.5 $\mu$ g of RNA was incubated with random hexamer primers $\left(3 \mu \mathrm{g} / \mu \mathrm{l}\right.$, Invitrogen) at $70{ }^{\circ} \mathrm{C}$ for $2 \mathrm{~min}$, then M-MLV-transcriptase (200U/ $\mu$ l, Invitrogen), RNase-Inhibitor $(40 \mathrm{U} / \mu \mathrm{l})$, DTT $(0.1 \mathrm{M}$, Invitrogen) and dNTP $(10 \mathrm{mM})$ was added. The reaction mix was incubated at $42{ }^{\circ} \mathrm{C}$ for $90 \mathrm{~min}$ and then $70{ }^{\circ} \mathrm{C}$ for $15 \mathrm{~min}$ for transcriptase deactivation. Real-time reverse-transcription polymerase chain reaction (RT-PCR) was performed using $5 \mu \mathrm{l}$ diluted cDNAs (1:200), $7 \mu \mathrm{l}$ SYBR green (Applied Biosystems, PowerSYBR green mix), $2 \mu$ l MAP2 primers (1.75 $\mu \mathrm{M}$, forward: 5'TCTAAAGAACATCCGTCACAGG3', reverse: 5'GGTGAGCATTGTCAAGTGAGC 3') or PPIA primers $(1.75 \mu \mathrm{M}$, forward: 5'TCCACTCCCAAGACTCAATC 3', reverse: $5^{\prime}$ CCATCGCTTCCACAATGTTC $3^{\prime}$ ) as the housekeeping gene. The reaction was performed in triplicate and StepOnePlus Real-time PCR System (Applied Biosystems) was used with the following conditions; 95 ${ }^{\circ} \mathrm{C}$ for $10 \mathrm{~min}$ (initial denaturation), 40 cycles at $95^{\circ} \mathrm{C}$ for $15 \mathrm{sec}$ and $60^{\circ} \mathrm{C}$ for $1 \mathrm{~min}$. A comparative threshold cycle method (2- $\Delta \Delta C \mathrm{CT})$ was used for the quantitation of the results.

\section{Statistical Analysis}

Statistical analyses were performed using Graphpad prism version 8 (La Jolla California USA). Mann-Whitney $U$ test was used and a result of $p<0.05$ was considered as statistically significant.

\section{Results}

After the re-analysis of human and mouse array CEL files, we identified differentially expressed genes of SMA samples when compared to their related control samples in both human and mouse datasets (ANOVA (eBayes) p-value $<0.05$ and fold change $<-1.5$ or fold change $>1.5$ ). In order to find expressional alterations of microtubule-related genes in SMA patients and SMA mice, we analyzed the expression of several transcripts, which are involved in both microtubule structure and the regulation of its dynamics. Differentially expressed gene lists of human and mouse datasets are given in Table I and II, respectively.

Among the microtubule-related genes that we analyzed in this study, 5 genes were differentially expressed in both the SMA patient and mouse model, while MAP2, MAP7 and TUBB4A showed similar gene expression alteration patterns (Figure 1A). The MAP2 gene was the only upregulated target, which drew our attention since the altered protein level of MAP2 was reported in a mouse model of amyotrophic lateral sclerosis (ALS), which is another motor neuron disease (31). Additionally, it has also been reported that either the protein level or the post-translational modifications of TAU, which is another microtubule-associated protein from the same protein family, was altered in ALS and SMA models, respectively $(14,21,31)$. Therefore, we subsequently focused on the MAP2 gene. To validate exon array results, we first used motor neuron-like NSC34 cells, which are murine neuroblastoma and spinal cord hybrid cell line as an in vitro model (32). We knocked down SMN by siRNA in the NSC34 cells and detected an upregulation in MAP2 gene expression by real time RT-PCR in SMNdepleted cells compared to scrambled controls (Figure 1B). Since the increase in gene expression was close to the significance level, we decided to analyze MAP2 gene expression in the severe SMA Taiwanese mouse model (22). First, we analyzed MAP2 gene expression in the total brain and spinal cord of late symptomatic p8 mice. We found a significant upregulation in both tissues of SMA mice compared to control littermates (Figure 1C). Considering that spinal cord motor neurons are primarily affected by SMN loss, we further analyzed MAP2 gene expression in the spinal cords of both pre-symptomatic (p1) and early symptomatic (p5) mice. A significant increase was detected in $\mathrm{p} 5$ but not p1 SMA mice (Figure 1C).

\section{Discussion}

SMA is a devastating genetic disease of childhood. Despite the recent therapeutic achievements with antisense oligonucleotide and successful clinical trials with gene therapy and small molecules, elucidating the functions of SMN protein and understanding the patho mechanisms of SMA is still needed. Re-analysis of public gene expression data is a promising tool to understand disease mechanisms. In this study, we re-analyzed raw data of previously published human exon-array and mouse microarray data that we obtained from the GEO database $(23,24)$ and specifically focused on genes regulating microtubule structure and/or function, since there is little knowledge about microtubule dynamics in SMA. Previously, an altered organization of microtubules in the presynaptic terminals of the axons innervating transverse abdominis of SMA mice have been reported in this commonly affected muscle (33). Additionally, stathmin, a microtubule depolymerizing protein, is upregulated in both SMN-depleted NSC34 cells and SMA mice and has 


\section{A}

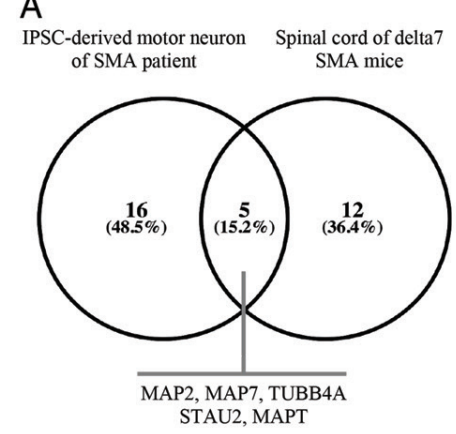

B

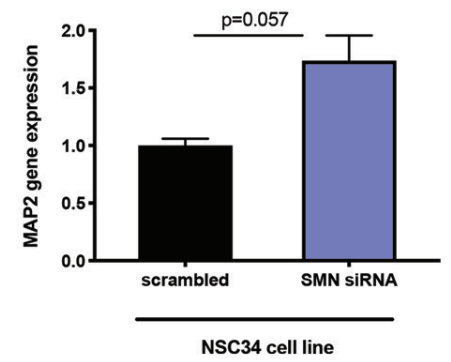

C

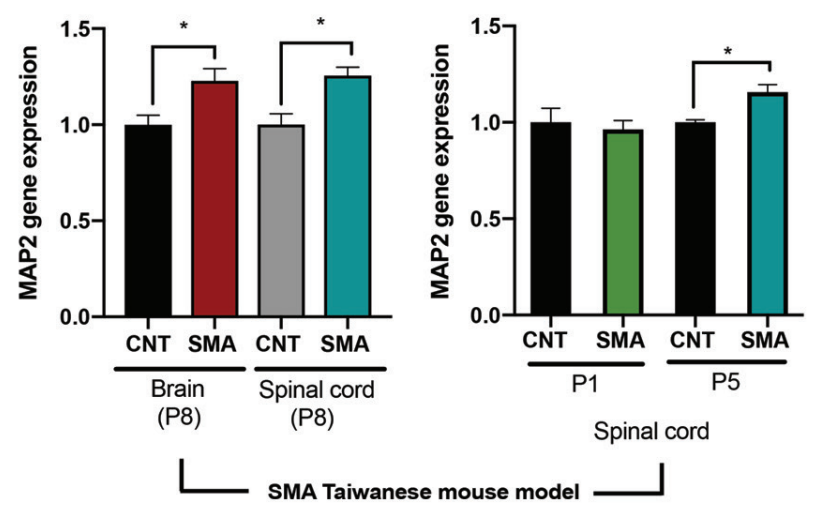

Figure 1. Expression analysis of genes regulating microtubule structure and its dynamics, A) Venn diagram of significantly altered genes in induced pluripotent stem cell-derived motor neurons of SMA patient and delta7 SMA mice using HuEx-1_0-st and Mouse430A_2 datasets, respectively. Fold changes of MAP2 transcript level in B) SMN knock down NSC34 cell line, $n=4$ biological replicates, C) spinal cord and brain tissues of p1, p5 and p8 severe SMA Taiwanese mouse model and heterozygous control littermates, for $p 1 ; n=5$ (control) and $n=4$ (SMA) mice, for $p 5$; $n=6$ (control) and $n=5$ (SMA) mice, for $p 8 ; n=5$ (control) and $n=5$ (SMA) mice. PPIA gene was used for normalization. Mann-Whitney $U$, * $p<0.05$, Data are presented as means with standard error of mean.

MAP: Microtubule-associated protein, SMN: Survival of motor neuron, SMA: Spinal muscular atrophy

Table I. Expressional alterations of microtubule-related genes in iPSC-derived motor neurons of SMA patient

\begin{tabular}{|c|c|c|c|c|c|}
\hline ID & $\begin{array}{l}\text { Fold } \\
\text { change }\end{array}$ & p-value & $\begin{array}{l}\text { FDR } \\
\text { p-value }\end{array}$ & Gene symbol & Description \\
\hline *3591459 & 2.24 & 0.0426 & 0.1914 & MAP1A; KRTAP6-1 & microtubule associated protein $1 \mathrm{~A}$; keratin associated protein 6-1 \\
\hline 3860229 & 1.71 & 0.001 & 0.0441 & CLIP3 & CAP-GLY domain containing linker protein 3 \\
\hline 2790823 & 1.61 & 0.021 & 0.1322 & MAP9 & microtubule-associated protein 9 \\
\hline *3784208 & 1.59 & 0.0151 & 0.1103 & DTNA; MAPRE2 & $\begin{array}{l}\text { dystrobrevin, alpha; microtubule-associated protein, RP/EB family, } \\
\text { member } 2\end{array}$ \\
\hline 2525533 & 1.56 & 0.006 & 0.0718 & MAP2 & microtubule associated protein 2 \\
\hline 3740126 & -1.5 & 0.0472 & 0.2023 & YWHAE; PAFAH1B1 & $\begin{array}{l}\text { tyrosine } 3 \text {-monooxygenase/tryptophan } 5 \text {-monooxygenase } \\
\text { activation protein, epsilon; platelet-activating factor } \\
\text { acetylhydrolase } 1 \mathrm{~b} \text {, regulatory subunit } 1 \text { (45kDa) }\end{array}$ \\
\hline 3526151 & -1.56 & 0.0258 & 0.1464 & TUBGCP3 & tubulin, gamma complex associated protein 3 \\
\hline 4002081 & -1.62 & 0.0034 & 0.0575 & MAP7D2 & MAP7 domain containing 2 \\
\hline 2654394 & -1.68 & 0.0467 & 0.2014 & FXR1 & fragile $X$ mental retardation, autosomal homolog 1 \\
\hline 3721926 & -1.73 & 0.0046 & 0.0642 & TUBG1 & tubulin, gamma 1 \\
\hline 3140640 & -1.75 & 0.0081 & 0.0823 & STAU2 & staufen double-stranded RNA binding protein 2 \\
\hline
\end{tabular}




\begin{tabular}{|c|c|c|c|c|c|}
\hline ID & $\begin{array}{l}\text { Fold } \\
\text { change }\end{array}$ & p-value & $\begin{array}{l}\text { FDR } \\
\text { p-value }\end{array}$ & Gene symbol & Description \\
\hline *3723687 & -1.87 & 0.0026 & 0.0524 & MAPT; MAPT-IT1 & microtubule associated protein tau; MAPT intronic transcript 1 \\
\hline 2901913 & -1.88 & 0.0022 & 0.0504 & TUBB & tubulin, beta class I \\
\hline 3764933 & -2.03 & 0.0193 & 0.1264 & TUBD1 & tubulin, delta 1 \\
\hline *4050485 & -2.05 & 0.0116 & 0.0968 & GRIN1; TUBB4B & $\begin{array}{l}\text { glutamate receptor, ionotropic, } \mathrm{N} \text {-methyl } \mathrm{D} \text {-aspartate } 1 \text {; tubulin, } \\
\text { beta } 4 \mathrm{~B} \text { class IVb }\end{array}$ \\
\hline 3847959 & -2.24 & 0.0012 & 0.0443 & TUBB4A & tubulin, beta 4 A class IVa \\
\hline 2600068 & -2.27 & 0.0026 & 0.0524 & TUBA4A & tubulin, alpha $4 a$ \\
\hline 2878662 & -2.51 & 0.0045 & 0.064 & DIAPH1 & diaphanous-related formin 1 \\
\hline 3515965 & -2.73 & 0.0071 & 0.0777 & DIAPH3 & diaphanous-related formin 3 \\
\hline 2975741 & -2.81 & 0.0002 & 0.0436 & MAP7 & microtubule-associated protein 7 \\
\hline *3453732 & -16.34 & 0.0447 & 0.1965 & $\begin{array}{l}\text { TUBA1B; LMBR1L; } \\
\text { TUBA1A }\end{array}$ & $\begin{array}{l}\text { tubulin, alpha 1b; limb development membrane protein 1-like; } \\
\text { tubulin, alpha 1a }\end{array}$ \\
\hline
\end{tabular}

${ }^{*}$ is used where the probe group is annotated to different gene symbols

been linked to defective microtubule polymerization (15). Hyperphosphorylation of TAU protein has been reported in the spinal cord of both SMA mice and patient samples (15). We observed an opposite gene expression profile of microtubule associated protein TAU (MAPT) between human and mouse gene expression results. According to our analysis, this target showed downregulation in human iPSC-derived motoneurons but it was upregulated in the spinal cords of SMA mice, which might be related to the presence of glial cells in the spinal cord (Table I and Table II). We focused on genes which have similar expression pattern in both arrays such as MAP2, MAP7 and TUBB4A. Among them, the MAP2 gene was the only upregulated one in both SMA patient and SMA delta 7 mice. It has been known that MAP2 plays a role on neuronal growth and degeneration (34). Considering its function in regulating microtubule stability in neurons and previous reports on gene expression alterations in ALS, we analyzed MAP2 gene expression for both in vitro and in vivo SMA model systems. Our experimental results were consistent with mouse microarray results, which showed a significant upregulation of MAP2 in the spinal cords of

Table II. Expressional alterations of microtubule-related genes in spinal cord of SMA delta 7 mice

\begin{tabular}{|l|l|l|l|l|l|}
\hline ID & $\begin{array}{l}\text { Fold } \\
\text { Change }\end{array}$ & p-value & FDR p-value & Gene Symbol & Description \\
\hline 1417885_at & 2.95 & $5.86 \mathrm{E}-06$ & 0.0002 & Mapt & microtubule-associated protein tau \\
\hline *144968__s_at & 2.87 & $4.93 \mathrm{E}-08$ & $5.60 \mathrm{E}-05$ & Tubb2a-ps2; Tubb2b & $\begin{array}{l}\text { tubulin, beta 2a, pseudogene 2; tubulin, beta 2B } \\
\text { class IIB }\end{array}$ \\
\hline 1424718_at & 2.67 & $4.80 \mathrm{E}-06$ & 0.0002 & Mapt & microtubule-associated protein tau \\
\hline 1421327_at & 2.42 & 0.0002 & 0.0019 & Map2 & microtubule-associated protein 2 \\
\hline 1424719_a_at & 2.41 & $1.13 \mathrm{E}-05$ & 0.0003 & Mapt & microtubule-associated protein tau \\
\hline 1450397_at & 2.07 & 0.0039 & 0.0166 & Map1b & microtubule-associated protein 1B \\
\hline 1421328_at & 2.04 & $6.44 \mathrm{E}-05$ & 0.0009 & Map2 & microtubule-associated protein 2 \\
\hline 1425534_at & 1.95 & $4.88 \mathrm{E}-05$ & 0.0008 & Stau2 & $\begin{array}{l}\text { staufen (RNA binding protein) homolog 2 } \\
\text { (Drosophila) }\end{array}$ \\
\hline 1452679_at & 1.88 & $7.85 E-06$ & 0.0003 & Tubb2b & tubulin, beta 2B class IIB \\
\hline 1418066_at & 1.79 & $1.01 E-05$ & 0.0003 & Cfl2 & cofilin 2, muscle \\
\hline 1428819_at & 1.77 & $7.75 E-05$ & 0.001 & Mapre1 & $\begin{array}{l}\text { microtubule-associated protein, RP/EB family, } \\
\text { member 1 }\end{array}$ \\
\hline
\end{tabular}




\begin{tabular}{|c|c|c|c|c|c|}
\hline ID & $\begin{array}{l}\text { Fold } \\
\text { Change }\end{array}$ & p-value & FDR p-value & Gene Symbol & Description \\
\hline 1415978_at & 1.71 & 0.0001 & 0.0016 & Tubb3 & tubulin, beta 3 class III \\
\hline 1425533_a_at & 1.67 & 0.0002 & 0.0019 & Stau2 & $\begin{array}{l}\text { staufen (RNA binding protein) homolog } 2 \\
\text { (Drosophila) }\end{array}$ \\
\hline 1416256_a_at & 1.59 & $1.44 \mathrm{E}-05$ & 0.0004 & Tubb5 & tubulin, beta 5 class I \\
\hline 1435347_at & 1.59 & $8.30 \mathrm{E}-05$ & 0.0011 & Stau1 & $\begin{array}{l}\text { staufen (RNA binding protein) homolog } 1 \\
\text { (Drosophila) }\end{array}$ \\
\hline 1422765_at & 1.53 & 0.0029 & 0.0133 & Mapre1 & $\begin{array}{l}\text { microtubule-associated protein, RP/EB family, } \\
\text { member } 1\end{array}$ \\
\hline 1424040_at & -1.56 & 0.0045 & 0.0186 & Map7d1 & MAP7 domain containing 1 \\
\hline 1450407_a_at & -1.64 & 0.0012 & 0.0071 & Anp32a & $\begin{array}{l}\text { acidic (leucine-rich) nuclear phosphoprotein } 32 \\
\text { family, member } A\end{array}$ \\
\hline 1418868_at & -1.67 & 0.0028 & 0.0128 & En2 & engrailed 2 \\
\hline 1426518_at & -1.74 & 2.01E-05 & 0.0004 & Tubgcp5 & tubulin, gamma complex associated protein 5 \\
\hline 1429894_a_at & -1.95 & $8.66 \mathrm{E}-05$ & 0.0011 & Map7 & microtubule-associated protein 7 \\
\hline 1423221_at & -2.08 & 0.0009 & 0.0056 & Tubb4a & tubulin, beta 4A class IVA \\
\hline 1421836_at & -2.79 & 0.0001 & 0.0014 & Map7 & microtubule-associated protein 7 \\
\hline 1421835_at & -3.21 & 0.0001 & 0.0013 & Map7 & microtubule-associated protein 7 \\
\hline 1460219_at & -4.39 & 0.0002 & 0.002 & Mag & myelin-associated glycoprotein \\
\hline
\end{tabular}

*is used where the probe group is annotated to different gene symbols

delta 7 SMA mice. We analyzed MAP2 gene expression in the brain and spinal cord tissues of the SMA Taiwanese mouse model and detected a significant increase in MAP2 gene expression in both tissues in the late symptomatic stage, which suggests a global differential expression of the MAP2 gene in the central nervous system. Results obtained from earlier developmental stages of SMA mice showed that MAP2 upregulation in the spinal cord occurs during the onset of disease symptoms. MAP2 induction may be a compensatory mechanism in an impaired cytoskeletal environment to maintain both microtubule stability and actin-microtubule crosslink during disease progression. Detailed studies on MAP2 expression in both neuronal and surrounding non-neuronal cells will help to reveal any functional consequences of this alteration.

\section{Conclusion}

Our findings may indicate an altered expression of the MAP2 gene during disease progression. Although our work is limited due to a lack of protein studies, our preliminary results indicate that microtubule regulatory proteins may be altered in SMN depleted cells. Further studies will be valuable in understanding the involvement of both MAP2 and other microtubule-related proteins to SMA patho mechanisms.

\section{Acknowledgement}

This study was supported by the Hacettepe University Scientific Research Projects Coordination Unit (International Collaboration Project Number: 13 G 602 003).

\section{Ethics}

Ethics Committee Approval: This article does not contain any studies with human participants performed by the authors.

Informed Consent: Informed consent wasn't obtained.

Peer-review: External and internal peer-reviewed.

\section{Authorship Contributions}

Concept: G.B., H.E.Y., Design: G.B., C.S., Data Collection or Processing: G.B., C.S., N.H., Analysis or Interpretation: G.B., C.S., N.H., P.C., H.E.Y., Literature Search: G.B., C.S., Writing: G.B., C.S., N.H., P.C., H.E.Y.

Conflict of Interest: The authors have no conflicts of interest to declare.

Financial Disclosure: This study was supported by the Hacettepe University Scientific Research Projects Coordination Unit (International Collaboration Project Number: 13 G 602 003). 


\section{References}

1. Sugarman EA, Nagan N, Zhu H, et al. Pan-ethnic carrier screening and prenatal diagnosis for spinal muscular atrophy: clinical laboratory analysis of $>72.400$ specimens. Eur I Hum Genet 2012;20:27-32.

2. Munsat TL, Davies KE. International SMA consortium meeting. (26-28 June 1992, Bonn, Germany). Neuromuscul Disord 1992;2:423-8.

3. Pearn J. Classification of spinal muscular atrophies. Lancet 1980;1:919-22.

4. Erdem H, Pehlivan S, Topaloglu H, Ozgüç M. Deletion analysis in Turkish patients with spinal muscular atrophy. Brain Dev 1999;21:86-9.

5. Lefebvre S, Burglen L, Reboullet S, et al. Identification and characterization of a spinal muscular atrophy-determining gene. Cell 1995;80:155-65.

6. Wirth B. An update of the mutation spectrum of the survival motor neuron gene (SMN1) in autosomal recessive spinal muscular atrophy (SMA). Hum Mutat 2000;15:228-37.

7. Bowerman $M$, Shafey $D$, Kothary R. Smn depletion alters profilin II expression and leads to upregulation of the RhoA/ ROCK pathway and defects in neuronal integrity. I Mol Neurosci 2007;32:120-31.

8. Hao le T, Duy PQ, Jontes JD, Wolman M, Granato M, Beattie CE. Temporal requirement for SMN in motoneuron development. Hum Mol Genet 2013;22: 2612-25.

9. McWhorter ML, Monani UR, Burghes AH, Beattie CE. Knockdown of the survival motor neuron (Smn) protein in zebrafish causes defects in motor axon outgrowth and pathfinding. I Cell Biol 2003;162:919-31.

10. Ohuchi K, Funato M, Kato Z, et al. Established stem cell model of spinal muscular atrophy is applicable in the evaluation of the efficacy of thyrotropin-releasing hormone analog. Stem Cells Transl Med 2016;5:152-63.

11. Rossoll W, Jablonka S, Andreassi C, et al. Smn, the spinal muscular atrophy-determining gene product, modulates axon growth and localization of beta-actin mRNA in growth cones of motoneurons. J Cell Biol 2003;163:801-12.

12. Hosseinibarkooie $S$, Peters $M$, Torres-Benito $L$, et al. The power of human protective modifiers: PLS3 and CORO1C unravel impaired endocytosis in spinal muscular atrophy and rescue SMA Phenotype. Am J Hum Genet 2016;99:647-65.

13. Nolle A, Zeug A, van Bergeijk J, et al. The spinal muscular atrophy disease protein $S M N$ is linked to the Rho-kinase pathway via profilin. Hum Mol Genet 2011;20:4865-78.

14. Miller $N$, Feng $Z$, Edens $B M$, et al. Non-aggregating tau phosphorylation by cyclin-dependent kinase 5 contributes to motor neuron degeneration in spinal muscular atrophy. I Neurosci 2015;35:6038-50.

15. Wen $\mathrm{HL}$, Lin $\mathrm{YT}$, Ting $\mathrm{CH}$, Lin-Chao $\mathrm{S}$, Li H, Hsieh-Li HM, et al. Stathmin, a microtubule-destabilizing protein, is dysregulated in spinal muscular atrophy. Hum Mol Genet 2010;19:1766-78.

16. Kou Y, Zhang S, Chen X, Hu S. Gene expression profile analysis of colorectal cancer to investigate potential mechanisms using bioinformatics. Onco Targets Ther 2015;8:745-52.
17. Grutzmann R, Boriss H, Ammerpohl O, et al. Meta-analysis of microarray data on pancreatic cancer defines a set of commonly dysregulated genes. Oncogene 2005;24:5079-88.

18. Wang X, Ning Y, Guo X. Integrative meta-analysis of differentially expressed genes in osteoarthritis using microarray technology. Mol Med Rep 2015;12:3439-45.

19. Su L, Wang C, Zheng C, Wei H, Song X. A meta-analysis of public microarray data identifies biological regulatory networks in Parkinson's disease. BMC Med Cenomics 2018;11:40.

20. Sucularli C, Shehwana H, Kuscu C, Dungul DC, Ozdag H, Konu O. Functionally conserved effects of rapamycin exposure on zebrafish. Mol Med Rep 2016;13:4421-30.

21. Dehmelt L, Halpain S. The MAP2/Tau family of microtubuleassociated proteins. Genome Biol 2005;6:204

22. Hsieh-Li HM, Chang JG, Jong YJ, et al. A mouse model for spinal muscular atrophy. Nat Genet 2000;24:66-70.

23. Edgar R, Domrachev M, Lash AE. Gene expression omnibus: NCBI gene expression and hybridization array data repository. Nucleic Acids Res 2002;30:207-10.

24. Barrett $\mathrm{T}$, Wilhite $\mathrm{SE}$, Ledoux $\mathrm{P}$, et al. NCBI GEO: archive for functional genomics data sets-update. Nucleic Acids Res 2013;41:991-5.

25. Corti S, Nizzardo M, Simone C, et al. Genetic correction of human induced pluripotent stem cells from patients with spinal muscular atrophy. Sci Transl Med 2012;4:165ra162.

26. Nizzardo $M$, Nardini $M$, Ronchi $D$, et al. Beta-lactam antibiotic offers neuroprotection in a spinal muscular atrophy model by multiple mechanisms. Exp Neurol 2011;229:214-25.

27. Bolstad BM, Irizarry RA, Astrand M, Speed TP. A comparison of normalization methods for high density oligonucleotide array data based on variance and bias. Bioinformatics 2003;19:185-93.

28. Irizarry RA, Hobbs B, Collin F, et al. Exploration, normalization, and summaries of high density oligonucleotide array probe level data. Biostatistics 2003;4:249-64.

29. Oliveros JC. Venny. An interactive tool for comparing lists with Venn's diagrams. http://bioinfogp.cnb.csic.es/tools/venny/ index.html. 2007-2015.

30. Hensel N, Ratzka A, Brinkmann H, Klimaschewski L, Grothe C, Claus $P$. Analysis of the fibroblast growth factor system reveals alterations in a mouse model of spinal muscular atrophy. PLoS One 2012;7:e31202.

31. Farah CA, Nguyen MD, Julien JP, Leclerc N. Altered levels and distribution of microtubule-associated proteins before disease onset in a mouse model of amyotrophic lateral sclerosis. I Neurochem 2003;84:77-86.

32. Cashman NR, Durham HD, Blusztajn JK, et al. Neuroblastoma $x$ spinal cord (NSC) hybrid cell lines resemble developing motor neurons. Dev Dyn 1992;194:209-21.

33. Torres-Benito L, Neher MF, Cano R, Ruiz R, Tabares L. SMN requirement for synaptic vesicle, active zone and microtubule postnatal organization in motor nerve terminals. PLoS One 2011;6:e26164.

34. Johnson GV, Jope RS. The role of microtubule-associated protein 2 (MAP-2) in neuronal growth, plasticity, and degeneration. ) Neurosci Res 1992;33:505-12. 